\title{
Efecto de las avenidas generadas después de eventos torrenciales extraordinarios sobre la salud mental de la población
}

\author{
Lucas Borja, P. , Lucas-Borja, M.E. ., , Gil Aguilar ,V., Fontalba Navas, A. ${ }^{3}$, \\ Pena Andreu J.M. ${ }^{4}$ \\ 'USMC-Almería, Hospital Torrecardenas, Sistema Andaluz de Salud. \\ ${ }^{2}$ Departamento de Ciencia y Tecnología Agroforestal y Genética, Universidad de Castilla La Mancha. \\ Campus Universitario s/n, CP 02071, Albacete
}

${ }^{3}$ Área Gestión Sanitaria Norte Almería, Servicio Andaluz de Salud, Consejería de Salud.

${ }^{4}$ Departamento Psiquiatría y Fisioterapia, Universidad de Málaga.

*e-mail: ManuelEsteban.Lucas@uclm.es

\begin{abstract}
Resumen
La restauración hidrológico-forestal ha tratado a lo largo de los años, disminuir los efectos negativos generados con la aparición de lluvias torrenciales mediante el uso de repoblaciones forestales, tratamientos selvícolas e hidrotecnias. Menos estudiados han sido los relacionados con la salud mental humana, los cuales se pueden hacer crónicos con el tiempo. El trastorno por estrés postraumático (TEPT) es una enfermedad que surge como respuesta tardía o diferida a un acontecimiento estresante o a una situación (breve o duradera) de naturaleza excepcionalmente amenazante o catastrófica. El objetivo del presente estudio es conocer la incidencia de TEPT en la población expuesta a un evento extraordinario de lluvias torrenciales y una gran avenida en el año 2012, concretamente en la comarca del noroeste de la provincia de Almería. En primer lugar se realizó un estudio de las precipitaciones extremas con ayuda de un SIG para el periodo de 1935 a 2012. Después se realizó un muestreo aleatorio simple sobre la población afectada y no afectada por las inundaciones. El análisis de datos pluviométricos demostró que la precipitación acontecida en 2012 fue extremadamente alta en comparación con los años anteriores. Los resultados también indican que existen fuertes relaciones entre el evento estudiado y la salud mental. Las diferencias en la aparición del TEPT están influenciadas por la edad, siendo las personas mayores las más afectadas después de una avenida. Del mismo modo, es necesario considerar fuentes de estrés secundario, como las pérdidas económicas para explicar los patrones de aparición de la enfermedad.
\end{abstract}

Palabras clave: Desastre natural, estrés post-traumático, inundación, precipitaciones extremas. 


\section{Introducción}

El número de investigaciones a nivel internacional sobre las consecuencias de los desastres naturales ha experimentado un auge en los últimos años, debido a las cuantiosas pérdidas materiales, sanitarias y humanas derivadas de estos fenómenos (Stanke et al., 2012; Kõlves et al., 2012). Sin embargo, hasta el momento hay escasa información al respecto en población española. Además, en el análisis de las consecuencias de un desastre natural frecuentemente se estima en costes económicos, obviando en muchos casos el impacto a largo plazo sobre la salud de la población.

Mientras que una proporción de las personas que sufren o se ven afectadas por la incidencia de desastres naturales se recuperan con el apoyo de sus familiares, amigos o compañeros, un gran número de ellas se ven afectadas en su salud, sus relaciones y su estado de bienestar. De hecho, se estima que en torno a un $22 \%$ pueden presentar problemas sociales y de salud mental, con tendencia a cronificarse en el tiempo (Stranke et al., 2012). Afectan a personas de todas las edades y pueden provocar: duelos, problemas económicos en las familias, trastornos de conducta en los niños, aumento de consumo de sustancias, aumento de la violencia doméstica, así como exacerbación, precipitación o provocación de enfermedades mentales (Kishore et al., 2008).

Las variaciones espaciales y temporales de las precipitaciones extremas se están viendo afectadas en el contexto actual de cambio climático. Se prevé que las variaciones de frecuencia e intensidad de las precipitaciones intensas en 24 horas favorecerán un incremento de los procesos torrenciales y geomorfológicos dinámicos, que provocarán un aumento de las pérdidas humanas y materiales. Las inundaciones son el desastre natural más frecuente. El impacto del cambio climático está incrementando el riesgo de ocurrencia de inundaciones a nivel mundial. En Europa 1,6 millones de habitantes cada año están en riesgo de sufrir una inundación (Jakubicka et al., 2010). La OMS estima que, en los últimos 10 años, las inundaciones en Europa han afectado a 3,4 millones de personas (World Health Organization, 2011). Al igual que en otros desastres naturales, las inundaciones repercuten gravemente sobre la salud, relaciones interpersonales y bienestar de la población. Sin embargo, se ha demostrado que en el caso de las inundaciones, estos daños tienen un período de recuperación posterior más largo que en otros desastres naturales, que incrementa el riesgo de estresares secundarios a largo plazo, como por ejemplo las dificultades económicas asociadas a la reconstrucción por la propia naturaleza del desastre. Además, entre las personas afectadas por una inundación suele existir un alto grado de miedo a que se repita el incidente.

A menudo, las experiencias vividas por las personas reflejan los significados personales y sociales del evento para ellos, y los significados que se derivan de ellas tienen más influencia en el impacto psicosocial que el propio evento en sí. La recuperación después de las inundaciones se caracteriza por la adaptación a las circunstancias que han cambiado y por la reconstrucción de las comunidades (Galea et al., 2007). Muchas personas experimentan angustia que puede ser relativamente transitoria después de cualquier desastre. Por otra parte, las experiencias de las personas que están en dificultades como consecuencia de las inundaciones no siempre son fáciles de distinguir de los síntomas de trastornos mentales comunes, lo que complica su diagnóstico. Por 
tanto, se sugiere que la incidencia de los trastornos mentales después de la inundación se puede incrementar considerablemente y que estos trastornos pueden persistir mucho después de que haya pasado la inundación. Esto subraya la importancia de la planificación y dar respuestas de salud y clínicas mentales públicas eficaces y oportunas.

El trastorno por estrés postraumático (TEPT) es una enfermedad que surge como una respuesta tardía o diferida a un acontecimiento estresante o a una situación (breve o duradera) de naturaleza excepcionalmente amenazante o catastrófica, que causarían por sí mismos malestar generalizado en casi todo el mundo (por ejemplo, catástrofes naturales o producidas por el hombre, combates, accidentes graves, el ser testigo de la muerte violenta de alguien, el ser víctima de tortura, terrorismo, de una violación o de otro crimen) (Montgomery et al., 2000).

Las características típicas del trastorno de estrés post-traumático son: episodios reiterados de volver a vivenciar el trauma en forma de re-experimentación o sueños que tienen lugar sobre un fondo persistente de una sensación de "entumecimiento" y embotamiento emocional, de despego de los demás, de falta de capacidad de respuesta al medio, de anhedonia y de evitación de actividades y situaciones evocadoras del trauma. Suelen temerse, e incluso evitarse, las situaciones que recuerdan o sugieren el trauma. En raras ocasiones pueden presentarse estallidos dramáticos y agudos de miedo, pánico o agresividad, desencadenados por estímulos que evocan un repentino recuerdo, una actualización del trauma o de la reacción original frente a él o ambos a la vez. Por lo general, hay un estado de hiperactividad vegetativa con hipervigilancia, un incremento de la reacción de sobresalto e insomnio. Los síntomas se acompañan de ansiedad y de depresión y es frecuente la ideación suicida. El consumo excesivo de sustancias psicotropas o alcohol puede ser un factor agravante (Spitzer et al., 1995). El comienzo sigue al trauma con un período de latencia cuya duración varía desde unas pocas semanas hasta meses (pero rara vez supera los seis meses).

El objetivo general de este trabajo es el de comparar la incidencia de TEPT y la evolución del estado de salud general entre una muestra de población adulta afectada por la inundación y una muestra de población control no afectada. Como objetivos específicos se presentan los siguientes:

- Estudiar la incidencia de TEPT en las dos poblaciones de estudio.

- Determinar la evolución del estado de salud en población afectada y control.

- Investigar la asociación entre exposición al evento extremo (inundación) y desarrollo de síntomas de TEPT y/o deterioro en salud general.

- Evaluar los factores sociodemográficos que actúen como modificadores de las asociaciones estudiadas.

\section{Material y métodos}

\subsection{Zona de estudio}

El pasado 28 de septiembre de 2012, la zona del Levante Almeriense quedó seriamente afectada tras la tormenta de viento, lluvia y granizo que se prolongó duran- 
te 45 minutos, con una precipitación entre los 70 y los 210 litros por metro cuadrado en algunas zonas. Los alcaldes de las zonas afectadas: Antas, Cuevas de Almanzora, Huércal-Overa, Mojacar, Pulpí y Vera publicaron a través de sus respectivas alcaldías un bando municipal en el que pidieron la declaración de zona catastrófica para los municipios. Entre los daños materiales ocasionados en la zona del Levante Almeriense por la inundación, el número de viviendas afectadas ascendió a 4.300, mientras que el número de vehículos que quedaron inutilizados se acerca a los 2.000, un número similar al de garajes quedaron cubiertos por el agua e inutilizables. Tres viaductos de la red viaria quedaron dañados mientras que la red municipal se vio afectada por acumulación de lodos y maleza. Como consecuencia de este desastre natural, diez personas perdieron la vida por las intensas lluvias en el sureste peninsular. Las pérdidas económicas derivadas de este desastre natural continúan en evaluación, aunque hasta el momento no se han estudiado las consecuencias del daño en la población sobre su salud o la aparición de secuelas a largo plazo, como el trastorno por estrés postraumático.

\subsection{Estudio de la precipitación extrema}

Dicho estudio ha consistido en el análisis de las precipitaciones históricas registradas en las estaciones meteorológicas de la zona de actuación y que son gestionadas por la agencia estatal de meteorología (AEMET). En concreto se ha utilizado una base de datos compuesta por datos pluviométricos de 35 estaciones meteorológicas repartidas por la zona de estudio. Los datos han correspondido a un periodo de 78 años (1935-2012), si bien es verdad que no todas las estaciones han presentado el mismo rango anual de datos. Para completar los años sin datos o estaciones sin valores registrados para determinados años se han realizado análisis de correlación entre estaciones cercanas.

Una vez realizada la exploración de los datos pluviométricos, se ha procedido a realizar una mapificación (representación espacial) de las precipitaciones acontecidas en el área de estudio. Se ha utilizado un Sistema de Información Geográfica (SIG) para distribuir espacialmente los datos de precipitación. Tomando como datos las precipitaciones recogidas en cada estación termo-pluviométrica, ha seguido los siguientes pasos:

1. Método de Interpolación de los datos de precipitación. Se utilizó el método de Distancia inversa ponderada (IDW) para interpolar las tendencias anuales de la variable climática analizada en cada estación. Este método de interpolación determinístico es uno de los más utilizados para la construcción de mapas e interpolación espacial (Díaz, 2008).

2. Verificación de la estadística básica de los datos obtenidos.

3. Obtención de un MDT a considerando como cota la precipitación.

4. Realización de las curvas de nivel (isolineas) a una cierta equidistancia.

5. Interpretación de los datos obtenidos a partir de las isolineas generadas con el SIG (López, 1995; Díaz, 2008). 


\subsection{Recogida de datos}

La recogida de datos la realizaron profesionales de Atención Primaria de los Centros de Salud de las áreas afectadas. Se eligió como lugar de muestreo los centros de salud y consultorios de Atención Primaria por la alta accesibilidad de los individuos de la población, existencia de una cobertura sanitaria del total de la población por el mismo sistema y por ser el primer punto de contacto cuando se detecta cualquier problema de salud, lo que hace que sea el sitio idóneo de implantación de herramientas de cribado, asumiendo que la prevalencia en población general puede ser ligeramente superior.

El seguimiento de los participantes en el estudio se realizó en Atención Primaria, dentro el mismo contexto y por el mismo profesional que participó en el reclutamiento, mediante cita concertada, la primera a los seis meses del reclutamiento y la segunda al año. Se registró el seguimiento y las visitas de forma anónima por el equipo de investigación mediante el código numérico asignado a cada participante. La evaluación consistió en un cuestionario sociodemográfico, la escala de salud general GHQ 12 y la escala de detección de Trastorno por Estrés Postraumático TQ. La escala GHQ-12 es un instrumento de cribado que tiene por objetivo detectar morbilidad psicológica y posibles casos de trastornos psiquiátricos en contextos como la atención primaria o en población general.

Para la ejecución de este trabajo se realizó un muestreo aleatorio simple en una población afectada por una inundación (Septiembre 2012) en el noreste de Almería (Número de sujetos seleccionados igual a 70). Se eligió una población control de un área cercana (distancia de $30 \mathrm{Km}$ y número de sujetos entrevistados igual a 91), con características sociodemográficas similares, no afectada por la inundación. La cohorte expuesta es una muestra representativa de la población afectada directa y seriamente por la inundación, corroborado por un estudio climatológico (municipios de Vera, Cuevas del Almanzora y Pulpí). La cohorte no expuesta corresponde a muestra representativa de población no afectada por la inundación (municipios de Zurgena, Macael y Albox). A todos los sujetos seleccionados para el estudio se les realizó un cuestionario socio-demográfico, cuestionario de Salud General (GHQ-12) y un cuestionario para valorar experiencias traumáticas (TQ). Se consideraron también distintos grados de afectación por la inundación (existencia o no de riesgo físico durante la inundación y / o pérdidas económicas debidas a ésta).

\subsection{Análisis de los datos}

El análisis del riesgo de presentar síntomas de TEPT se estimó mediante regresión logística, y los predictores de las puntuaciones TQ mediante un modelo de regresión lineal multivariante. La variables que se han estudiado se dividen en variables dependientes e independientes. La variables dependientes fueron:

- Aparición de Trastorno por Estrés Postraumático, evaluada mediante el instrumento de cribado de TEPT, la escala TQ (11)

- Deterioro en salud general, medida por la escala GHQ-12 (12) 
Variables independientes fueron:

— Variables socio-demográficas que pueden influir en la aparición de estrés postraumático:

- Sexo (variable cualitativa de dos categorias: hombre-mujer).

- Edad (variable cuantitativa calculada en años 18-99).

- Nivel de estudios (variable cualitativa de cinco categorias: básicos, bachillerato, diplomado, licenciado, doctorado).

- Ocupación (variable cualitativa de cinco categorias: asalariado cuenta ajena, asalariado cuenta propia, ama de casa, desempleado, jubilado).

- Nivel de afectación por la inundación (variable cuantitativa: medido en daños o pérdidas materiales valorados en euros, aportada por peritaje).

- Lugar de residencia detectado por sistemas de información geográfica (SIG).

\section{Resultados y discusión}

Con el objetivo de conseguir una potencia del $90 \%$ para detectar diferencias mediante una prueba Chi cuadrado bilateral para dos muestras independientes, teniendo en cuenta que el nivel de significación es del 5\%, y asumiendo que la proporción en el grupo expuesto es del $22 \%$ (11 sujetos), la proporción en el grupo no expuesto es del 1,95\% (13 sujetos), y que el tamaño de cada cohorte es el mismo fue necesario incluir 53 sujetos en cada grupo, totalizando 106 personas en el estudio.

Una vez realizado el estudio de las precipitaciones medias anuales acontecidas en la zona, se pueden observar diferentes periodos en los que se supera de forma significativa la precipitación media acontecida en la zona de estudio para los últimos 77 años.

Tabla 1. Comparación entre la precipitación media anual para el 2012 y el periodo 1935-2012.

\begin{tabular}{l|c|c|c|c}
\hline & \multicolumn{2}{|c|}{ Coordenadas UTM } & \multicolumn{2}{c}{ Precipitación media anual (mm) } \\
\hline Estación meteorológica & $\mathrm{X}$ & $\mathrm{Y}$ & $\mathrm{P}(1935-2012)$ & $\mathrm{P} 2012$ \\
\hline ALBOX & 575103 & 4140325 & 245,45 & 259,08 \\
\hline CARBONERAS & 598250 & 4095134 & 221,26 & 375,31 \\
\hline CARBONERAS & & & & \\
(CENTRAL TERMICA) 6332I & 597701 & 4093371 & 191,23 & 259,26 \\
\hline CARBONERAS,C.TÉRM. 6332 X & 597604 & 4093216 & 133,64 & 100,78 \\
\hline CHIRIVEL C H SUR 7187A & 565009 & 4161327 & 338,91 & 602,05 \\
\hline CHIRIVEL (C H SEGURA) 7187 & 564519 & 4161293 & 289,18 & 422,25 \\
\hline CUEVAS DE ALMANZORA & 599294 & 4128500 & 214,11 & 407,98 \\
\hline GARRUCHA FARO 6340 & 604316 & 4114753 & 240,98 & 377,07 \\
\hline GARRUCHA FARO 6340X & 605047 & 4115440 & 270,63 & 408,04 \\
\hline GERGAL (CMA) & 541039 & 4108289 & 254,24 & 340,46 \\
\hline GERGAL A NACIMIENTO & 535624 & 4104937 & 195,39 & 297,20 \\
\hline HUERCAL OVERA 6367B & 593515 & 4138790 & 258,78 & 387,29 \\
\hline
\end{tabular}




\begin{tabular}{|c|c|c|c|c|}
\hline \multirow[b]{2}{*}{ HUERCAL OVERA 6367} & \multicolumn{2}{|c|}{ Coordenadas UTM } & \multicolumn{2}{|c|}{ Precipitación media anual $(\mathrm{mm})$} \\
\hline & 593142 & 4139218 & 312,63 & 461,00 \\
\hline $\begin{array}{l}\text { HUERCAL OVERA } \\
\text { (CUESTA COLORADA) (CMA) } 6369\end{array}$ & 590793 & 4135801 & 262,82 & 413,27 \\
\hline HUERCAL OVERA (EL PEÑASCAL) & 592620 & 4133047 & 258,49 & 413,27 \\
\hline $\begin{array}{l}\text { HUERCAL OVERA } \\
\text { (INST.LABORAL) 6367A }\end{array}$ & 594016 & 4137995 & 253,72 & 405,84 \\
\hline HUERCAL OVERA (LOS ORIVES) 6368 & 595293 & 4133910 & 241,71 & 373,52 \\
\hline $\begin{array}{l}\text { HUERCAL OVERA } \\
\text { (PUERTECICO PERELLON)(CMA) 6365E }\end{array}$ & 589655 & 4150122 & 265,51 & 407,61 \\
\hline HUERCAL OVERA LAS PERNERAS 6366E & 590528 & 4137617 & 194,36 & 280,68 \\
\hline $\begin{array}{l}\text { HUERCAL OVERA-PUERTO LUMBRERAS } \\
\text { (C.P.C.) } 6371\end{array}$ & 598825 & 4147297 & 189,64 & 280,68 \\
\hline LOS GALLARDOS (CMA) & 594036 & 4114262 & 315,70 & 487,54 \\
\hline LUBRIN-EL HOYO & 582889 & 4119076 & 368,15 & 534,41 \\
\hline MACAEL (CMA) & 561597 & 4131837 & 411,24 & 563,25 \\
\hline MOJACAR & 601967 & 4111118 & 318,36 & 430,41 \\
\hline PULPI CANALEJAS (CMA) & 610469 & 4140045 & 243,63 & 365,45 \\
\hline SERÓN, ESTACIÓN & 542635 & 4134184 & 320,86 & 421,09 \\
\hline SORBAS & 577833 & 4106083 & 308,99 & 498,32 \\
\hline TABERNAS (PLANTA SOLAR) & 556409 & 4105389 & 218,54 & 348,79 \\
\hline TABERNAS A SORBAS C P C (CMA) & 562660 & 4104724 & 233,66 & 319,50 \\
\hline VELEZ RUBIO (C H SEGURA) & 581430 & 4167390 & 281,93 & 570,63 \\
\hline VELEZ RUBIO I L & 581256 & 4167666 & 330,69 & 521,82 \\
\hline VELEZ RUBIO TONOSA & 584541 & 4162798 & 345,77 & 550,79 \\
\hline VERA (INSTITUTO LABORAL) & 600842 & 4122663 & 257,35 & 416,20 \\
\hline VERA LAS ALGARROBINAS & 603783 & 4123931 & 295,13 & 454,11 \\
\hline ZURGENA & 584962 & 4133428 & 228,30 & 360,34 \\
\hline ZURGENA FUENTE DEL PINO & 580022 & 4127678 & 244,74 & 416,28 \\
\hline
\end{tabular}

Las precipitaciones registradas en el año 2012 superan con creces la media de las precipitaciones anuales acontecidas en la zona de estudio dentro del periodo 19352012. Por ejemplo, la precipitación que se registro en la estación de Huercal Overa (código 6367) en 2012 fue de $461 \mathrm{~mm}$ anuales, mientras que la media para esa estación es de $312 \mathrm{~mm}$ anuales. Del mismo modo para la población de Vera (Estación las Algarrobinas) en 2012 se ha registrado una precipitación anual de $454 \mathrm{~mm}$, mientras que la media para todos los años en los que existen datos es de $295 \mathrm{~mm}$. Por lo tanto y a partir de los análisis realizados, se puede apuntar que el año 2012 constituye un año excepcional en lo que a cantidad de precipitación se refiere. Esta información puede verse en las Fig. 1 y 2 , en las que se representa un modelo digital del terreno con la variable precipitación media anual representada en cada mapa. La figura 1 muestra las precipitaciones medias anuales en la zona desde 1935 hasta 2012 mien- 
tras que la figura 2 muestra las precipitaciones medias anuales en la zona de estudio para el año 2012. Las diferencias son importantes y como resultado de ello, las inundaciones que acontecieron en la zona de estudio durante septiembre de 2012.

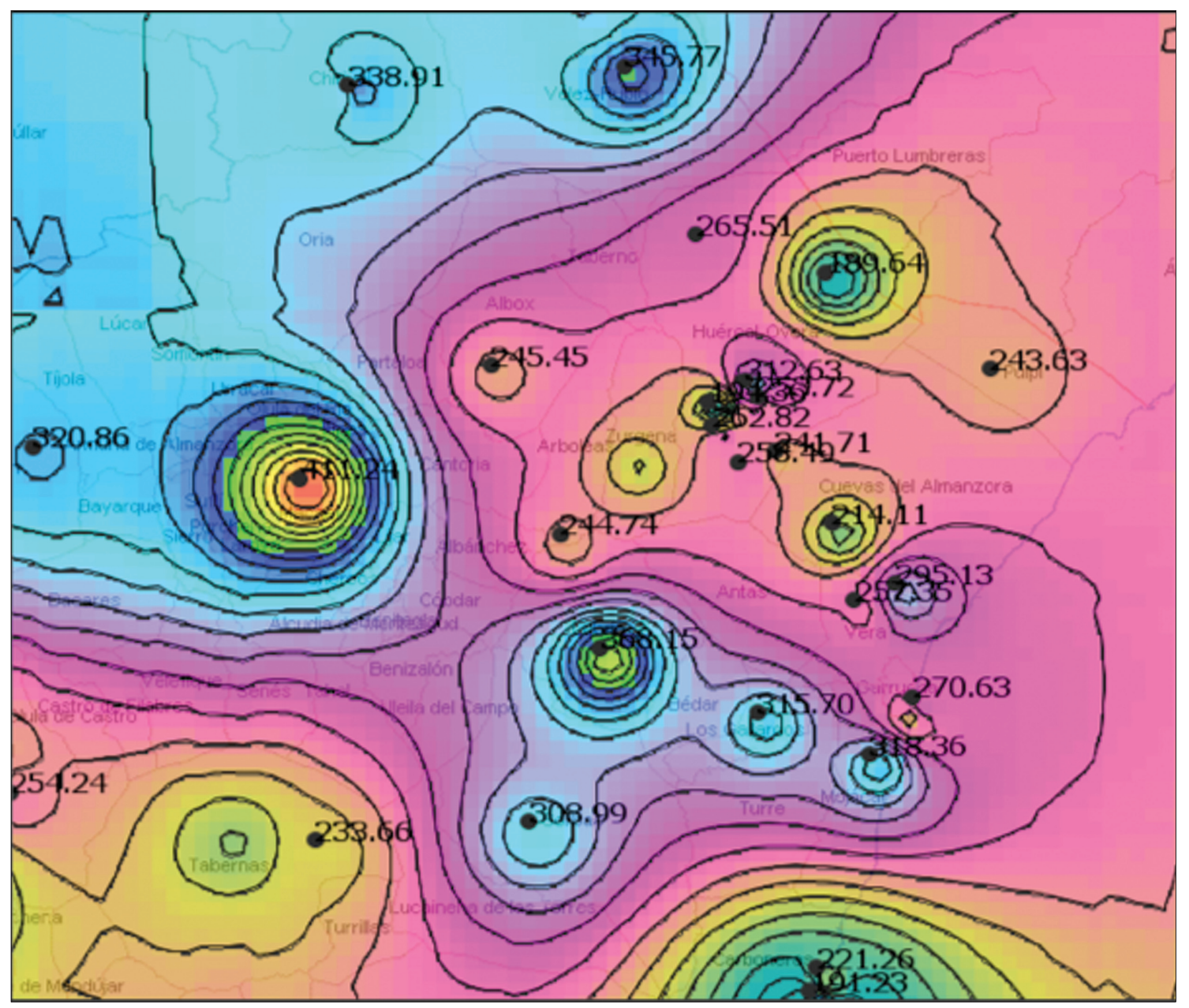

Figura 1. Imagen ráster que representa las precipitaciones medias anuales en la zona de estudio desde 1935 hasta 2012.

Las características de ambas poblaciones de estudio (afectadas y no afectadas por la inundación) se resumen en la Tab. 1. No se encontraron diferencias estadísticamente significativas en edad y sexo entre ambos grupos. El riesgo de presentar síntomas de TEPT (odds ratio) fue 8,18 veces superior (IC 95\% 3,99-17,59) en la zona afectada con respecto a la no afectada. Para estudiar los factores potencialmente predictores de las puntuaciones TQ se construyó un modelo lineal multivariante incluyendo todas las variables independientes en población afectada (edad ,sexo, riesgo físico en la inundación, pérdidas económicas o daños materiales), siendo el haber sufrido pérdidas materiales la única variable explicativa del modelo que mostró una asociación estadísticamente significativa (Tab. 2). Además, dentro de la población afectada por la inundación, la gente de mayor edad fue más proclive a desarrollar tras- 




Figura 2. Imagen ráster que representa las precipitaciones medias anuales en la zona de estudio para el año 2012.

torno por estrés postraumático, si bien es verdad que la diferencia no fue estadísticamente significativa.

Tabla 2. Características descriptivas de la población estudiada

\begin{tabular}{l|c|c|c|}
\hline & AFECTADA & NO AFECTADA & P-Valor \\
\hline $\mathrm{N}$ & 70 & 91 & \\
\hline Edad media (desv. típica) & $51,58(15,42)$ & $51,74(17,45)$ & 0,95 \\
\hline$\%$ mujeres & 64,03 & 62,60 & 0,82 \\
\hline Puntuación media escala TQ (desv. típica) & $5,39(6,02)$ & $1,59(4,13)$ & $<0,01 *$ \\
\hline$\%$ en riesgo físico inundación & 12,90 & 2,20 & $<0,01 *$ \\
\hline$\%$ con pérdidas materiales inundación & 47,10 & 3,30 & $<0,01 *$ \\
\hline
\end{tabular}

Ante un evento torrencial extraordinario y la consecuente avenida o inundación, la persona sufre de estrés en múltiples frentes, no sólo como se produce la inunda 
Tabla 3. Predictores de las puntuaciones en la escala de detección de Trastorno por Estrés Postraumático TQ. Modelo de regresión lineal multivariante.

\begin{tabular}{l|c|c|c|c|}
\hline & Parámetro & Error estándar & T-valor & P-Valor \\
\hline (Intercept) & 2,18 & 1,69 & 1,29 & 0,19 \\
\hline Edad & $-0,02$ & 0,02 & $-0,84$ & 0,10 \\
\hline Sexo & 1,05 & 0,90 & 1,17 & 0,24 \\
\hline Riesgo físico & 2,75 & 1,48 & 1,84 & 0,06 \\
\hline Pérdidas económicas & 7,24 & 0,97 & 7,41 & $0,01 *$ \\
\hline
\end{tabular}

ción, sino también en el período de anticipación antes de una inundación y durante la fase de limpieza y recuperación (Stanke et al., 2012; Kõlves et al., 2012). Además, las personas afectadas por las inundaciones pueden experimentar una serie de emociones, incluyendo la ansiedad, el miedo, la ira, la frustración, la tristeza y la pena (Jakubicka et al., 2010). Por lo tanto, es natural que las personas que experimentan eventos traumáticos, como las inundaciones, a experimentar dificultad para dormir, pérdida de apetito, estados de ánimo deprimidos o enojados y los sentimientos más elevados de ansiedad. Todas estas circunstancias y problemas, han sido reflejados en los cuestionarios planteados por los encargados de recoger la información, si bien es verdad, que la población afectada por la inundación sobrepaso con creces, lo niveles de la población control. Además, las pérdidas materiales y el hecho de tener que hacer frente a importantes pérdidas económicas, ha desencadenado problemas mentales y la aparición de estrés post-traumático en la población afectada por la inundación. De hecho, la variable económica, es la única que ha resultado ser significativa después de los análisis estadísticos realizados. Por lo tanto, habría que tener en cuenta que este trastorno psicológico, encuadrado dentro del grupo de los trastornos de ansiedad, estaría relacionado con las pérdidas económicas antes que con la edad o el sexo de las personas afectadas.

\section{Conclusiones}

El presente trabajo ha puesto de manifiesto que las personas afectadas por inundaciones pueden ver gravemente afectada su salud mental. En cualquier estudio epidemiológico pueden existir dificultades a la hora de determinar el área de afectación geográfica de un desastre natural para cuantificar su repercusión. Esta dificultad es salvable mediante un estudio preciso de la zona afectada a través de un mapa que delimite geográfica y demográficamente la zona en la que incide la catástrofe natural. De igual manera, las consecuencias de la catástrofe natural sobre la población están relacionadas tanto con la intensidad y nivel de afectación por la misma así como con factores sociodemográficos como los incluidos en el estudio, lo cual abre una línea innovadora, en tanto en cuanto no existen apenas investigaciones que vinculen la intensidad del estresor en catástrofes naturales de carácter hídrico con la intensidad de 
la respuesta (psicológica y física) en población humana. Ajustando la población afectada por edad y sexo, aparece como variable principal identificativa de la aparición de TEPT las pérdidas económicas realcionadas con el desastre, más que variables de exposición directa como sería estar en riesgo físico. Estos resultados son plausibles con la idea en epidemiología de considerar las carencias económicas como factor de riesgo de enfermedades tanto físicas como mentales. Dado que además el TEPT puede aparecer en población general, la comparación con una población control de carácterísticas similares refuerza esta asociación. Al tratarse de un estudio de cohortes hay que contemplar la dificultad en el reclutamiento y seguimiento posterior de los sujetos. Por otro lado, se parte de un sistema sanitario único y universal que atiende a esta población en concreto, con una alta accesibilidad al centro de salud y un seguimiento muy estrecho de los casos por parte del médico de Atención Primaria, lo que minimizará las pérdidas. La población atendida en Atención Primaria a priori no tiene por qué ser idéntica a la población general en cualquier medio, pero en el modelo asistencial de la Comunidad Autónoma Andaluza sí es representativa por la cobertura sanitaria total, y es el medio ideal para el desarrollo de cribado de cualquier enfermedad en población general, como se ha justificado previamente. Bajo el punto de vista de la Salud Pública, la exploración de los datos pluviométricos, podría convertirse en un magnífico predictor (sencillo, objetivo y mensurable) del nivel de respuesta sanitaria que la sociedad debe plantear para situaciones futuras. En virtud de estos resultados podemos concluir que las graves inundaciones en la zona Norte de Almería de Septiembre de 2012 han causado un deterioro de la salud de la población afectada, así como un aumento de la incidencia de síntomas de Trastorno por Estrés Postraumático.

\section{Bibliografía}

Bobes, J., Calcedo-Barba, A., García, M., Francois, M., Rico-Villademoros, F., González, M.P., Bascarán, M.T., Bousoño, M., 2000. Evaluación de las propiedades psicométricas de la versión española de cinco cuestionarios para la evaluación del trastorno de estrés postraumático. Actas Esp Psiquiatr. 28(4), 207-218.

Díaz, 2008. Interpolación espacial de la precipitación pluvial en la zona de Barlovento y Sotavento del Golfo de México. Instituto Nacional de Investigaciones Forestales, Agrícolas y Pecuarias Texcoco, México.

Du, W., FitzGerald, G.J., Clark, M., Hou, X.Y., 2010. Health impacts of floods. Prehosp Disaster Med. 25(3), 265-7.

Galea, S., Brewin, C.R., Gruber, M., Jones, R.T., King, D.W., King, L.A., 2007. Exposure to hurricane-related stressors and mental illness after Hurricane Katrina. Archives of General Psychiatry. 64(12), 1427-1434.

Gamble, J.L., Hurley, B.J., Schultz, P.A., Jaglom, W.S., Krishnan, N., Harris, M. 2012. Climate Change and Older Americans: State of the Science. Environ Health Perspect. 121(1), 15-22.

Jakubicka, T., Vos, F., Phalkey, R., Marx, M. 2010. Health impacts of flood in Europe: data 
gaps and information needs from a spatial perspective. A microdis reperotd: Brussels: Centre for Research on the Epidemiolgy of Disasters (CRED).

Kishore, V., Theall, K.P., Robinson, W., Pichon, J., Scribner, R., Roberson, E., 2008. Resource loss, coping, alcohol use, and posttraumatic stress symptoms among survivors of Hurricane Katrina: a cross- sectional study. Amer J Disaster Med. 3(6), 345-357.

Kõlves, K., Kõlves, K.E., De, Leo., 2013. Natural disasters and suicidal behaviours: A systematic literature review. J Affect Disord. 146,1-14.

López V. 1995. Comparación de 4 métodos numéricos utilizados en la interpolación puntual de precipitación pluvial en el estado de Veracruz, México. Tesina de Maestría. 71 p.

Montgomery, S., Bech, P., Angst, J., Davidson, J.R.T., Delini-Stula, A., Van Ree, J.M., Van Zwieten-Boot, B.J., Zohar, J., Dunbar, G., Montgomery, D., Wakelin, J., 2000. Post traumatic stress disorder: guidelines for investigating efficacy of pharmacological intervention. European Neuropsychopharmacology. 10, 297-303

Paranjothy, S., Gallacher, J., Amlôt, R., Rubin, G.J., Page, L., Baxter, T., Wight, J., Kirrage, D., McNaught, R., Palmer, S.R., 2011. Psychosocial impact of the summer 2007 floods in England. BMC Public Health. 3, 11-145.

Peek-Asa, C., Ramirez, M., Young, T., Cao, Y., 2012. Flood-Related Work Disruption and Poor Health Outcomes Among University Students. Prehosp Disaster Med. 3,1-6.

Powers, J.R., Loxton, D., Baker, J., Rich, J.L., Dobson, A.J., 2012. Empirical evidence suggests adverse climate events have not affected Australian women's health and well-being. Public Health. 36(5), 452-7.

Richardson, J., Coid, J., Petruckevitch, A., Chung, W., Moorey, S., Feder, G., 2002. Identifying domestic violence: cross sectional study in primary care. BMJ. 324-274.

Sánchez-López, M. P., Dresch, V., 2008. The 12-Item General Health Questionnaire (GHQ12): reliability, external validity and factor structure in the Spanish population. Psicothema. 8, 8-20.

Spitzer, R.L., Kroenke, K., Linzer, M., Hahn, S.R., Williams, J.B., de Gruy, F.V., Brody, D., Davies, M., 1995. Health-related quality of life in primary care patients with mental disorders: results from the PRIME-MD Study. JAMA.

Stanke. C., Murray, V., Amilot, R., Nurse, J., Williams, R., 2012. The effect of flooding on mental health: outcomes and recommendations from a review of the literature. PLOS Current disaster.

World Health Organization, 2011. Climate change: Facts and figures. Regional Office for Europe. 